\title{
Hypoxic-immune Model Based on Lung Squamous Cell Carcinoma Reveals the Effects of Inflammation and Hypoxia on Drug Resistance, CD8 Cell Depletion and Prognosis
}

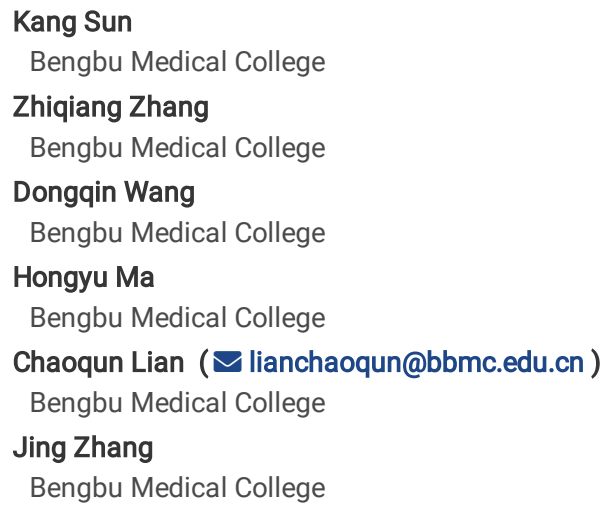




\section{Abstract}

Background: Lung squamous cell carcinoma (LUSC) is a malignant tumor with high mortality and poor prognosis. More evidence shows that hypoxia and the immune environment play an essential role in cancer progression, but the specific impact on lung squamous cell carcinoma is unclear. This study mainly establishes immune and hypoxia risk models to predict the prognosis of patients and formulates personalized treatment plans for patients according to the specific conditions of hypoxia regulation and immune invasion in high-risk groups.

Results: Based on the combined use of multiple data, 380 hypoxia and immune co-related genes (HMGs) were obtained $\rrbracket$ to establish the risk model of immune and hypoxia. Through the use of comprehensive analysis methods, the model has a high predictive value. The survival rate of the high-risk group is low, and the CD8-T cell depletion factor is widely distributed in high-risk groups. It has a large number of neutrophils and low CD8 cells. In addition, hypoxia, inflammation, and drug resistance-related pathways are also abundant in high-risk groups. We also found that high-risk patients were generally resistant to chemotherapeutic drugs. Finally, we constructed a competing endogenous RNA (CeRNA) network closely related to risk genes, including 9 mRNAs, 10 MicroRNAs (miRNAs), and 16 long non-coding RNAs (IncRNAs).

Conclusions:This study specifically analyzed the effects of hypoxia regulation and immune Infiltration on the prognosis of patients. It provided a new idea for patients to improve the prognosis and personalized treatment.

\section{Introduction}

Lung squamous cell carcinoma is a common malignant tumor with high mortality, accounting for about 30\% [1-2] of lung cancer. Although significant progress has been made in cancer treatment, lung cancer patients' average 5-year survival rate is less than $18 \%$ [3]. The reasons may be the limitations of molecular targeted therapy and cytotoxic chemotherapy drug resistance to patients [4-6]. However, immune checkpoint programmed death 1 (PD-1) / programmed death-ligand 1 (PD-L1) is blocked by immune checkpoint block (ICB). Therapy has made a significant breakthrough in improving the prognosis of patients, but only $20 \%$ of patients will obtain the therapeutic effect. Some ICB treatments will also hurt the prognosis of patients [7-10], including immunerelated inflammatory reactions and cytokines, which can promote tumor progression and immune escape. [11-13] Interestingly, hypoxia is closely related to the formation of inflammatory environment, tumor development, and drug resistance [13-17]. Therefore, establishing and screening immune hypoxia co-related risk models and prognostic markers will play an essential role in improving patients' prognoses and personalized treatment.

In this study, the immune and hypoxia-related risk model established by us has a high ability to predict the prognosis of patients. The high-risk group is significantly enriched in hypoxia, inflammation, drug resistance signal pathways, many inflammatory cells, the CD8 cell depletion factor, and low CD8 cells. This phenomenon reveals that hypoxia regulation and immune invasion have an essential impact on patients' prognosis and provide a new thinking scheme for the treatment of patients in the future.

\section{Methods}

\section{Data acquisition and preprocessing}

We downloaded RNA sequencing (RNA-Seq) gene expression data of 49 normal groups and 502 tumor groups from the Cancer Genome Atlas ( TCGA) (https://portal.gdc.cancer.GOv), a variety of corresponding clinicopathological information, including age, gender, survival time, survival status, T stage, $\mathrm{M}$ stage, Nstage, TMNstage, At the same time, from the Gene Expression Omnibus (GEO) (https://www.ncbi.nlm.nih.GOv/GEO). Then, based on the HALLMARK data set in gene set enrichment analysis (GSEA), I downloaded 200 genes related to the hypoxia pathway. From the TIMER (The Tumor Immune Estimation Resource) website (http://cistrome.dfci.harvard.edu/TIMER ), We downloaded 1650 immune function genes.

We used the string (functional protein binding network) tool to construct the PPI network of 200 hypoxia-related genes; its purpose is to find out the core hypoxia gene.

\section{Screening of the co-related genes of immunity and hypoxia (HMCORS)}

We used the "limma" package in R language to analyze the differences between normal and tumor groups. The screening criteria were | logFC | > 2 and FDR $<0.05$. At the same time, the correlation of significantly different genes, immune function genes, and hypoxia core genes were analyzed, respectively. The threshold of screening was the correlation coefficient $(|\operatorname{cor}|<0.4$, p-value $<0.05)$. The intersection of immune and hypoxia-related genes was obtained

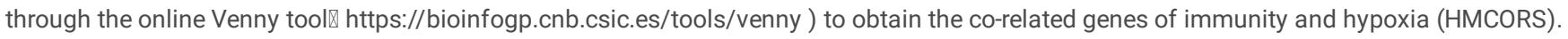

\section{KEGG and GO functional enrichment analysis}

The annotation, visualization, and integrated discovery (David) performed KEGG and GO functional enrichment analysis for immune hypoxia co-related genes.

\section{Establishment and validation of immune and hypoxia-related risk models}

The most significant prognostic genome was first screened by univariate Cox regression analysis and the minor absolute shrinkage and selector operator (lasso). Then the risk coefficient of each sample was calculated according to the risk gene expression and multivariate Cox regression analysis. The risk score was calculated as follows: risk core $=$ risk coefficient 1 * gene expression $1+\ldots$ risk coefficient $\mathrm{N}$ * gene expression $\mathrm{N}$. The high and low-risk groups are divided according to the median value of the risk score. The risk groups in the risk model established by the GEO data set are divided according to the optimal detection thresholds. Kaplan Meier (KM) survival analysis was performed for high and low-risk groups, and receiver operating characteristics (ROC) curves were used to evaluate the predictive value of the risk model for three years $(1,3$, and 5 years).

Page 2/15 
Firstly, univariate and multivariate Cox regression analysis evaluated the risk score and multiple pathological and clinical factors. Then, the nomogram was constructed according to the risk score and numerous clinical characteristics (age, gender, T, M, N, and TMNstage) as prognostic factors. The calibration chart was used to evaluate the stability of the predictive ability of the nomogram.

\section{Survival analysis of high and low-risk groups under different clinicopathological groups}

Based on the "survival" package in R language, the clinicopathological parameters were divided into age $<=65$, age $>65, \mathrm{MALE}, \mathrm{FEMALE}, \mathrm{M0}$, M1, N0-1, N2-3, T1-2, T3-4, stage-II, and stagellI-IV groups. Then the survival of KM was analyzed in different clinicopathological groups.

\section{Scoring of Immune cell infiltration in different risk groups}

CIBERSOFT calculates the composition ratio of 22 immune cells in vivo based on the deconvolution alGOrithm and the gene expression in each sample (18). We downloaded the immune score of tcga-LUSC samples calculated, based on the CIBERSOFT method, from the TIMER database (tumor immune estimation resource). At the same time, we used the "e1071", "parallel," and "preprocessor" packages in the R tool to calculate the immune cell ratio of LUSC samples in the GEO database. The standard value of screening is p-value $<0.05$. At the same time, we used the "e1071", "parallel," and "preprocessor" packages in the R tool to calculate the immune cell ratio of different risk groups of LUSC in the GEO database. The standard value of screening is p-value < 0.05 . Finally, we analyzed the immune scores and immune cell infiltration of different risk groups according to the "ggpubr" package in the $\mathrm{R}$ language.

\section{Gene set variation analysis (GSVA)}

The "GSVA" package of the R tool and multiple data sets (GO, KEGG, HALLMARK) in the gene enrichment analysis tool (GSEA) was used to analyze the enrichment pathways of different risk groups. $\mathrm{P}<0.05$ was considered statistically significant.

\section{Prediction of chemosensitivity of patients in different risk groups}

We use the "pRRophetic" package in R language to predict the half-maximal antibiotic concentration (IC50) of patients in different risk groups for chemotherapeutic drugs to infer patients' drug resistance in high-risk and low-risk groups. The primary method is to use the genetics of drug sensitivity in Cancer (GDSC) (www.cancerrxgene.org/) cell line expression Spectrum, TCGA and GEO gene expression profiles were used to construct ridge regression model, and then pRRophetic method in R package was used to calculate IC50 of chemotherapy drugs

\section{Establishment of CeRNA network}

the encyclopedia of rna interactomes (ENCORI) and microRNA target prediction database (miRDB) were used to score the corresponding target genes mRNA, miRNA, and IncRNA of risk genes. Then, correlation analysis and survival analysis were carried out for risk genes and predicted target genes. The screening criteria were the default settings in each online website, with correlation coefficient $(C O R<-0.1)$ and $p$-value $<0.05$.

\section{Immune and hypoxia co-related genes}

Through the difference analysis between normal and tumor groups, 3632 genes with a significant difference were obtained. Then, the PPI network of 200 hypoxia pathway genes was constructed by using a string database, and then 49 immune core genes with the highest degree of protein interaction were screened. Cytoscape software (Cytoscape 3.7.1) was used Mapping. Draw the correlation between all hypoxia core genes Matrix heat map. The correlation between immune function genes and hypoxia core genes and differentially expressed genes were analyzed, respectively, and 399 hypoxia-related genes (HPCORS) and 1750 immune-related genes (IMCORS) were obtained. Finally, we used Venny online software (Venny 2.1.0) to get the intersection of immune and hypoxia-related genes and draw the Wayne diagram. Finally, 380 immune and hypoxia-related genes (HMCORS) were obtained.

\section{Go, and KEGG functional enrichment analysis was performed on HMCORS}

Online David analysis was performed to analyze the go and KEGG function enrichment of $380 \mathrm{HMGs}$. We found that these HMCORS were mainly enriched in the immune and hypoxia-related pathways of going analysis, such as response to hypoxia, transforming growth factor, beta activated receptor activity, blood vessel matching, regulation of immune response, vasculogenesis, Negative regulation of cytokine secret, immune response, changing growth factor-beta binding, leukotriene production involved in inflammatory response and gluconeogenesis. The KEGG pathways of immunity and hypoxia mainly include glycolysis/gluconeogenesis, Salmonella infection, ECM receptor interaction, and the HIF-1 signaling pathway.

\section{Establish and predict immune and hypoxia-related risk models}

Univariate Cox regression analysis was performed on 380 HMCORS, and 44 prognostic genes were screened. Then, Lasso regression analysis was used to select the best prognostic gene. Then the risk score of each sample was calculated through multivariate Cox regression analysis, as follows: risk score $=$ $(0.143$ * PTGIS expression $)+(0.413$ * C10orf55 expression $)+(0.046$ * NAPSA $)+(0.142$ *MYADM $)$, To visualize the difference between the two groups, we drew PCA principal component analysis. Secondly, the prognosis of the high-risk group in TCGA and GEO data sets was significantly worse than that of the low-risk group. ROC curve analysis showed that the AUC values in 1,3 , and 5 years of the model were 0.635 respectively, $0.681,0.631$. The correlation between four risk genes and risk scores was also analyzed. It was found that PTGIS, C10orf55, NAPSA, and MYADM were highly distributed in the high-risk group and positively correlated with the risk score.

Effects of risk model and clinicopathological parameters on the prognosis of patients 
To further understand the impact of clinicopathological factors and risk models on the prognosis of patients, we found that risk score was the most significant independent factor through univariate and multivariate Cox regression analysis. At the same time, In these pathological groups, we found that the prognosis of high-risk group was worse than that of low-risk group, Including age $>65(P=0.003), M a l(P<0.001), M 0(P<0.001), N 0-1(P=0.012), N 2-3(P=$ 0.001), T1-2 ( $\mathrm{P}=0.001)$, stage $\mathrm{I}-\mathrm{II}(\mathrm{P}<0.001)$.

\section{Establishment and evaluation of prognostic nomogram}

The risk score and a variety of clinicopathological parameters were used as prognostic factors to establish a nomogram. The calibration map showed that the nomogram showed better prediction accuracy than the ideal model compared with a perfect model. The c-index of the nomogram for OS prediction was 0.652 .

\section{Immune cell infiltration landscape in different risk groups}

To further explore the potential mechanism of the model in immunity, the CIBERSOFT algorithm is used to analyze the distribution level of immune cells and scores in high-risk and low-risk groups. We found that macrophages M0, CD4 memory resting, neutrophils, immunity, and matrix scoring are mainly distributed in high-risk groups in the TCGA data set, while B cells memory, B cells naive, T cells CD8 are spread primarily on low-risk groups; at the same time, using Rpackage spearman correlation analysis, the risk score was negatively correlated with B cells naive, $T$ cells CD8, $T$ cells folgular helper and dendritic cells resting, which was opposite to macrophages M0, CD4 memory resting, neutrophils, immune and matrix scores. Tox, PD1, CTLA4, BTL, LAG3, TIM3, MYADM, TGFB1 immune-related factors were higher in the high-risk group. In the GEO dataset, high-risk groups were mainly distributed in macrophages M1, neutrophils, T cells gamma delta, immune and matrix scores. Low-risk groups were primarily distributed in plasma cells, T cells CD8, T cells CD4 naïve, and NK cells resting. Risk scores were positively correlated with B cells naive, T cells CD4 memory resting, T cells gamma delta, neutrophils, eosinophil, immune and matrix scores, In contrast to T cells CD4 naive, T cells CD8 and NK cells resting, CTLA4, BTL, Lag3, tim3, MYADM, and TGFB1 immune-related factors were highly expressed in high-risk groups.

\section{GSVA revealed that the high-risk group was closely related to immune response and hypoxia regulation}

We performed gene set variation analysis (GSA) on tcga-LUSC RNA Seq data and GEO gene expression data, based on various data sets in GSEA (GO, KEGG, HALLMARK). The GSVA results of the two databases showed that 31, 34, and 19 related functional pathway genes were enriched in high-risk groups, and 10, 7, and 9 immune and hypoxia-related signal pathways were distributed in high-risk groups, respectively (Table 1). Finally, based on different data sets (go, KEGG, HALLMARK), we found that these hypoxia and immune-related pathways were highly distributed in the high-risk group and positively correlated with the risk score.

\section{Sensitivity of patients in high and low-risk groups to chemotherapy drugs}

To further explore the drug resistance of different risk groups, we used a prrophic algorithm to calculate the $50 \%$ inhibitory concentration (IC50) of various conventional chemotherapy drugs (bleomycin, cisplatin, docetaxel, doxorubicin, etoposide, gefitinib, gemcitabine, and paclitaxel) in patients in high-risk and low-risk groups. In the TCGA training set, doxorubicin, etoposide, and gefitinib had higher IC50 in high-risk groups. In the GEO test set, cisplatin, etoposide, and gefitinib had higher IC50 in the high-risk group.

\section{Establishment and validation of risk genes related CeRNA network}

We used the online analysis websites ENCORI (the Encyclopedia of RNA interactomes) and m miRDB (microRNA target prediction database) to analyze four risk gene-related target genes (mRNA, miRNA, IncRNA). Then, based on KM survival analysis and spersman correlation analysis, The target genes opposite to the correlation of risk genes and the survival analysis results were screened. The network diagram and Sankey diagram of Cerna of risk genes and target genes were constructed by Cytoscape software and "ggalluvial" in R language.

\section{Results}

\section{Immune and hypoxia co-related genes}

The difference analysis between normal and tumor groups obtained 3632 genes with a significant difference (Fig. 1A-B). Then, the PPI network of 200 hypoxia pathway genes was constructed by using a string database, and then 49 immune core genes with the highest degree of protein interaction were screened (Fig. 1C). Cytoscape software (Cytoscape 3.7.1) was used Mapping. Draw the correlation between all hypoxia core genes Matrix heat map (Fig. 1D). The correlation between immune function genes and hypoxia core genes and differentially expressed genes were analyzed, respectively, and 399 hypoxia-related genes (HPCORS) and 1750 immune-related genes (IMCORS) were obtained. Finally, we used Venny online software (Venny 2.1.0) to get the intersection of immune and hypoxia-related genes and draw the Wayne diagram (Fig. 1E). Finally, 380 immune and hypoxia-related genes (HMCORS) were obtained.

\section{Go, and KEGG functional enrichment analysis was performed on HMCORS}

Online David analysis was performed to analyze the go and KEGG function enrichment of $380 \mathrm{HMGs}$. We found that these HMCORS were mainly enriched in the immune and hypoxia-related pathways of going analysis, such as response to hypoxia, transforming growth factor, beta activated receptor activity, blood vessel matching, regulation of immune response, vasculogenesis, Negative regulation of cytokine secret, immune response, changing growth factor-beta binding, leukotriene production involved in inflammatory response and gluconeogenesis. The KEGG pathways of immunity and hypoxia mainly include glycolysis/gluconeogenesis, Salmonella infection, ECM receptor interaction, and the HIF-1 signaling pathway (Fig. 1F). 
Univariate Cox regression analysis was performed on 380 HMCORS, and 44 prognostic genes were screened (Fig. 2A). Then, Lasso regression analysis was used to select the best prognostic gene (Fig. 2B-C). Then the risk score of each sample was calculated through multivariate Cox regression analysis, as follows: risk score $=(0.143$ * PTGIS expression $)+(0.413 *$ C10orf55 expression $)+(0.046$ * NAPSA $)+(0.142$ *MYADM $)$ (Fig. 2D), To visualize the difference between the two groups, we drew PCA principal component analysis (Fig. 2E). Secondly, the prognosis of the high-risk group in TCGA and GEO data sets was significantly worse than that of the low-risk group (Fig. 2F). ROC curve analysis showed that the AUC values in 1, 3, and 5 years of the model were 0.635 (Fig. $2 \mathrm{G})$, respectively, $0.681,0.631$. The correlation between four risk genes and risk

\section{Protein-protein interactive (PPI) network analysis}

scores was also analyzed. It was found that PTGIS, C10orf55, NAPSA, and MYADM were highly distributed in the high-risk group and positively correlated with the risk score (Fig. $2 \mathrm{H}-\mathrm{J})$.

\section{Effects of risk model and clinicopathological parameters on the prognosis of patients}

To further understand the impact of clinicopathological factors and risk models on the prognosis of patients, we found that risk score was the most significant independent factor through univariate and multivariate Cox regression analysis (Fig. $3 \mathrm{~A}-\mathrm{C})$. At the same time, In these pathological groups, we found that the prognosis of high-risk group was worse than that of low-risk group, Including age $>65(P=0.003), M a l(P<0.001), M 0(P<0.001)$, N0-1 $(P=$ 0.012), N2-3 ( $P=0.001)$, T1-2 $(P=0.001)$, stage $\mathrm{III}(\mathrm{P}<0.001)$ (Fig. $3 \mathrm{H})$.

\section{Establishment and evaluation of prognostic nomogram}

The risk score and various clinicopathological parameters were used as prognostic factors to establish a nomogram (Fig. 3D). The calibration map showed that the nomogram showed better prediction accuracy than the ideal model compared with a perfect model (Fig. 3E-G). The c-index of the nomogram for OS prediction was 0.652 .

\section{Immune cell infiltration landscape in different risk groups}

To further explore the potential mechanism of the model in immunity, the CIBERSOFT algorithm is used to analyze the distribution level of immune cells and scores in high-risk and low-risk groups. We found that macrophages M0, CD4 memory resting, neutrophils, immunity, and matrix scoring are mainly distributed in high-risk groups in the TCGA data set, while B cells memory, B cells naive, T cells CD8 are spread primarily on low-risk groups; at the same time, using Rpackage spearman correlation analysis, the risk score was negatively correlated with B cells naive, $T$ cells CD8, T cells folgular helper and dendritic cells resting, which was opposite to macrophages M0, CD4 memory resting, neutrophils, immune and matrix scores (Fig. 4A, C). Tox, PD1, CTLA4, BTL, LAG3, TIM3, MYADM, TGFB1 immune-related factors were higher in the high-risk group (Fig. 4B). In the GEO dataset, The prognosis of the high-risk group was worse than that of the low-risk group (Fig. 5A). Then, based on the CIBERSOFT algorithm, high-risk groups were mainly distributed in macrophages M1, neutrophils, $\mathrm{T}$ cells gamma delta, immune and matrix scores. Low-risk groups were primarily distributed in plasma cells, T cells CD8, T cells CD4 naïve, and NK cells resting. Risk scores were positively correlated with B cells naive, T cells CD4 memory resting, T cells gamma delta, neutrophils, eosinophil, immune and matrix scores, In contrast to T cells CD4 naive, T cells CD8 and NK cells resting (Fig. 5B-C, E). CTLA4, BTL, Lag3, tim3, MYADM, and TGFB1 immune-related factors were highly expressed in high-risk groups (Fig. 5D).

\section{GSVA revealed that the high-risk group was closely related to immune response and hypoxia regulation}

We performed gene set variation analysis (GSA) on tcga-LUSC RNA Seq data and GEO gene expression data, based on various data sets in GSEA (GO, KEGG, HALLMARK). The GSVA results of the two databases showed that 31,34, and 19 related functional pathway genes were enriched in high-risk groups (Fig. $6 \mathrm{~A}$, D, G), and 10, 7, and 9 immune and hypoxia-related signal pathways were distributed in high-risk groups, respectively (Table 1). Finally, based on different data sets (go, KEGG, HALLMARK), we found that these hypoxia and immune-related pathways were highly distributed in the high-risk group and positively correlated with the risk score(Fig. 5B-C, E-F, H-I).

\section{Sensitivity of patients in high and low-risk groups to chemotherapy drugs}

To further explore the drug resistance of different risk groups, we used a prrophic algorithm to calculate the IC50 of various conventional chemotherapy drugs (bleomycin, cisplatin, docetaxel, doxorubicin, etoposide, gefitinib, gemcitabine, and paclitaxel) in patients in high-risk and low-risk groups. In the TCGA training set, doxorubicin, etoposide, and gefitinib had higher IC50 in high-risk groups. In the GEO test set, cisplatin, etoposide, and gefitinib had higher IC50 in the highrisk group (Fig. 7A-G).

\section{Establishment and validation of risk genes related CeRNA network}

We used the online analysis websites ENCORI (the Encyclopedia of RNA interactomes) and m miRDB (microRNA target prediction database) to analyze four risk gene-related target genes (mRNA, miRNA, IncRNA). Then, based on KM survival analysis and spersman correlation analysis, The target genes opposite to the correlation of risk genes and the survival analysis results were screened (Fig. 8C) (Fig. 9A-C). The network diagram and Sankey diagram of Cerna of risk genes and target genes were constructed by Cytoscape software and "ggalluvial" in R language (Fig. 8A-B).

\section{Discussion}


Hypoxia is an inherent feature of most solid tumors, not an accident [19-20]. In recent years, more and more reports have clarified that hypoxia is closely related to tumor generation, angiogenesis, cancer cell metastasis, and drug resistance [21-24]. At the same time, immune cells in the tumor microenvironment play an essential role in tumorigenesis and progression [25-27]. Interestingly, in recent studies, we found that immunity and immune regulation of the tumor microenvironment are closely related [28-30]. Still, at present, In lung squamous cell carcinoma, there is an interaction between hypoxia regulation and immune invasion, and its impact on the prognosis of patients is unclear.

In this study, we made the intersection of immune and hypoxia-related genes to obtain immune and hypoxia-related genes (HMCORS). Then, based on a single factor, multifactor and lasso Cox regression analysis, and immune hypoxia risk model was established, including four risk genes (PTGIS, C10orf55, NAPSA, and MYADM). The high expression of PTGIS can promote tumor-associated macrophages (TAMs) and T-regulatory cells (Tregs), and it is unfavorable to the prognosis of patients with lung cancer, gastric cancer, and ovarian cancer [31]. C10orff has few research reports on cancer at present. In this study, the prognosis of patients with lung squamous cell carcinoma with high expression of C10orf55 is poor. In lung cancer, the expression of NAPSA is closely related to the formation of inflammatory tumor lymphocytes (TIL) and inflammatory immune environment [32]. The expression of MYADM may significantly impact the prognosis of prostate cancer patients [33]. The prognostic model based on these four genes is the most significant independent prognostic factor compared with other clinicopathological factors. Through nomogram, calibration chart, ROC curve, and two data sets (TCGA, GEO), The application of the model show that the model has high, stable, and accurate prognosis prediction ability. The survival difference analysis is made in different clinicopathological groups and high-risk and low-risk groups. It is found that the patients in the high-risk group have poor prognosis under age greater than 65, Male, M0, T1-2, N01, N2-3, and stagel-II groups, which provides new thinking for the selection of clinical scheme. Then, the CIBERSOFT algorithm was used to analyze the immune cell invasion of TCGA training and GEO test sets. We found that the distribution of neutrophils in the high-risk group was higher, and the distribution of CD8 cells in the low-risk group was higher.

At the same time, the immune score and matrix score of the high-risk group were higher than those of the low-risk group. Using three data sets in GSEA (GO, KEGG, HALLMARK), Gsva analysis of the high-risk group showed that the high-risk group was mainly enriched in hypoxia, inflammatory response, inflammatory cells, and TGFB related signal pathway. The inflammatory response in acute diseases often promotes the occurrence and transformation of various cancers, and neutrophils play a significant role in inflammatory response [34-35]. Interestingly, in chronic inflammatory and tumor diseases, hypoxia and inflammatory reactions often occur together and affect each other [36-39]. In discussing drug resistance in different risk groups, the prrophetic algorithm calculates the IC50 of chemotherapeutic drugs from LUSC samples of two data sets. We found that the IC50 values of etoposide and gefitinib are generally higher in high-risk groups. Similarly, In recent studies, many reports have shown that hypoxia and inflammatory response can increase the drug resistance of tumors to etoposide and gefitinib [40-44], which further illustrates the stability and accuracy of the model and detection method. While CD8 cell-related depletion factors (MYADM, BTLA, CTLA4, Lag3, and tim3) are generally elevated in high-risk groups [45-52], Other kinds of lite have also reported that hypoxia and chronic inflammatory response can accelerate the depletion of CD8 cells [53-54], TGFB1 can also inhibit the related functions of CD8 cells and promote tumor metastasis [55].

\section{Conclusion}

In conclusion, the immune hypoxia risk model established by us can accurately and stably predict the prognosis of patients. The distribution level of hypoxia and inflammatory response in the high-risk group is high, accompanied by increased CD8 cell depletion factor and drug resistance. These reactions may have a significant impact on the prognosis of patients and put forward essential thoughts for personalized treatment of patients

\section{Abbreviations}




\begin{tabular}{|c|c|}
\hline Abbreviations & Corresponding notes \\
\hline LUSC & Lung squamous cell carcinoma \\
\hline HMGs & hypoxia-related genes \\
\hline CeRNA & competing endogenous RNA. \\
\hline IncRNAs & long non-coding RNAs \\
\hline miRNA & MicroRNA \\
\hline mRNA & Coding RNA \\
\hline PD-1 & programmed death 1 \\
\hline PD-L1 & programmed death-ligand 1 \\
\hline ICB & immune checkpoint block \\
\hline RNA-Seq & RNA sequencing \\
\hline PPI & Protein-protein interactive network analysis \\
\hline GSVA & gene set variation analysis \\
\hline IC50 & $50 \%$ inhibitory concentration \\
\hline TCGA & the Cancer Genome Atlas \\
\hline GEO & Gene Expression Omnibus \\
\hline lasso & the minor absolute shrinkage and selector operator \\
\hline KM & Kaplan Meier \\
\hline ROC & receiver operating characteristics \\
\hline GSEA & enrichment analysis tool \\
\hline GDSC & the genetics of drug sensitivity in Cancer cell \\
\hline ENCORI & the encyclopedia of rna interactomes \\
\hline COR & correlation coefficient \\
\hline IMCORS & immune-related genes \\
\hline TAMs & tumor-associated macrophages \\
\hline Tregs & T-regulatory cells \\
\hline TIL & inflammatory tumor lymphocytes \\
\hline
\end{tabular}

\section{Declarations}

\section{Consent for publication}

All relevant authors agree to publish

\section{Author's contribution}

$\mathrm{KS}, \mathrm{ZZ}$ and DW provide the idea and design of this article. Clinical data were collected and analyzed by KS, ZZ, and DW. KS and HM drafted the first draft of the article and the drawing of charts. CL and JZ reviewed the revised paper. All authors read and approved the final manuscript

\section{Author details}

${ }^{1}$ Research Center of Clinical Laboratory Science, Bengbu Medical College, Bengbu 233030, China; ${ }^{2}$ Department of Biochemistry and Molecular Biology, School of Laboratory Medicine, Bengbu Medical College, Bengbu 233030, China; ${ }^{3}$ Department of Genetics, School of Life Sciences, Bengbu Medical College, Bengbu 233000, China; ${ }^{4}$ Department of Clinical Medicine, Bengbu Medical College, Bengbu 233030, China;

\section{Competing interests}

The authors declare that they have no competing interests.

\section{Availability of data and materials}

All data generated or analyzed during this study are included in this published article. 


\section{FUNDING}

This study was supported by grants from the National Natural Science Foundation of China (No. 81672314), the Key Natural Science Project of Anhui Provincial Education Department (No.KJ2015A289, KJ2018A0221, and KJ2020A0578) and National Innovation Program for College Students (Nos. 201910367041 and 202010367008$)$.

\section{References}

1. Bray F, Ferlay J, Soerjomataram I, Siegel RL, Torre LA, Jemal A Global cancer statistics 2018: GLOBOCAN estimates of incidence and mortality worldwide for 36 cancers in 185 countries. CA: a cancer journal for clinicians. 2018;68(6):394-424.

2. Travis WD, Brambilla E, Nicholson AG, Yatabe Y, Austin JHM, Beasley MB, Chirieac LR, Dacic S, Duhig E, Flieder DB et al The 2015 World Health Organization Classification of Lung Tumors: Impact of Genetic, Clinical and Radiologic Advances Since the 2004 Classification. Journal of thoracic oncology : official publication of the International Association for the Study of Lung Cancer. 2015;10(9):1243-1260.

3. Chien CM, Yang JC, Wu PH, Wu CY, Chen GY, Wu YC, Chou CK, Tseng CH, Chen YL, Wang LF et al Phytochemical naphtho[1,2-b] furan-4,5-dione induced topoisomerase II-mediated DNA damage response in human non-small-cell lung cancer. Phytomedicine : international journal of phytotherapy and phytopharmacology. 2019;54(unknow):109-119.

4. Kelly RL, Le D, Zhao J, Wittrup KD Reduction of Nonspecificity Motifs in Synthetic Antibody Libraries. Journal of molecular biology. 2018;430(1):119-130.

5. Brahmer J, Reckamp KL, Baas P, Crinò L, Eberhardt WE, Poddubskaya E, Antonia S, Pluzanski A, Vokes EE, Holgado E et a/ Nivolumab versus Docetaxel in Advanced Squamous-Cell Non-Small-Cell Lung Cancer. The New England journal of medicine. 2015;373(2):123-135.

6. Jordan EJ, Kim HR, Arcila ME, Barron D, Chakravarty D, Gao J, Chang MT, Ni A, Kundra R, Jonsson P et a/ Prospective Comprehensive Molecular Characterization of Lung Adenocarcinomas for Efficient Patient Matching to Approved and Emerging Therapies. Cancer discovery. 2017;7(6):596-609.

7. Andrews LP, Yano H, Vignali DAA Inhibitory receptors and ligands beyond PD-1, PD-L1 and CTLA-4: breakthroughs or backups. Nature immunology. 2019;20(11):1425-1434.

8. Topalian SL, Hodi FS, Brahmer JR, Gettinger SN, Smith DC, McDermott DF, Powderly JD, Sosman JA, Atkins MB, Leming PD et al Five-Year Survival and Correlates Among Patients With Advanced Melanoma, Renal Cell Carcinoma, or Non-Small Cell Lung Cancer Treated With Nivolumab. JAMA oncology. 2019;5(10):1411-1420.

9. Malhotra J, Jabbour SK, Aisner J Current state of immunotherapy for non-small cell lung cancer. Translational lung cancer research. 2017;6(2):196-211.

10. Kim Y, Kim CH, Lee HY, Lee SH, Kim HS, Lee S, Cha H, Hong S, Kim K, Seo SW et al Comprehensive Clinical and Genetic Characterization of Hyperprogression Based on Volumetry in Advanced Non-Small Cell Lung Cancer Treated With Immune Checkpoint Inhibitor. Journal of thoracic oncology : official publication of the International Association for the Study of Lung Cancer. 2019;14(9):1608-1618.

11. Jing B, Wang T, Sun B, Xu J, Xu D, Liao Y, Song H, Guo W, Li K, Hu M et al IL6/STAT3 Signaling Orchestrates Premetastatic Niche Formation and Immunosuppressive Traits in Lung. Cancer research. 2020;80(4):784-797.

12. Liu S, Ye D, Wang T, Guo W, Song H, Liao Y, Xu D, Zhu H, Zhang Z, Deng J Repression of GPRC5A is associated with activated STAT3, which contributes to tumor progression of head and neck squamous cell carcinoma. Cancer cell international. 2017;17(unknow):34.

13. Hanahan D, Weinberg RA Hallmarks of cancer: the next generation. Cell. 2011;144(5):646-674.

14. Taylor CT, Colgan SP Regulation of immunity and inflammation by hypoxia in immunological niches. Nature reviews Immunology. 2017;17(12):774-785.

15. Eltzschig HK, Carmeliet P Hypoxia and inflammation. The New England journal of medicine. 2011;364(7):656-665.

16. Song X, Feng L, Liang C, Yang K, Liu Z Ultrasound Triggered Tumor Oxygenation with Oxygen-Shuttle Nanoperfluorocarbon to Overcome HypoxiaAssociated Resistance in Cancer Therapies. Nano letters. 2016;16(10):6145-6153.

17. Sahu A, Kwon I, Tae G Improving cancer therapy through the nanomaterials-assisted alleviation of hypoxia. Biomaterials. 2020;228(unknow):119578.

18. Newman AM, Liu CL, Green MR, Gentles AJ, Feng W, Xu Y, Hoang CD, Diehn M, Alizadeh AA Robust enumeration of cell subsets from tissue expression profiles. Nature methods. 2015;12(5):453-457.

19. Gray LH, Conger AD, Ebert M, Hornsey S, Scott OC The concentration of oxygen dissolved in tissues at the time of irradiation as a factor in radiotherapy. The British journal of radiology. 1953;26(312):638-648.

20. Mathieu J, Zhang Z, Zhou W, Wang AJ, Heddleston JM, Pinna CM, Hubaud A, Stadler B, Choi M, Bar M et al HIF induces human embryonic stem cell markers in cancer cells. Cancer research. 2011;71(13):4640-4652.

21. Nishida N, Kudo M Oxidative stress and epigenetic instability in human hepatocarcinogenesis. Digestive diseases (Basel, Switzerland). 2013;31(5-6):447453.

22. Wu XZ, Xie GR, Chen D Hypoxia and hepatocellular carcinoma: The therapeutic target for hepatocellular carcinoma. Journal of gastroenterology and hepatology. 2007;22(8):1178-1182

23. Semenza GL HIF-1: upstream and downstream of cancer metabolism. Current opinion in genetics \& development. 2010;20(1):51-56.

24. Semenza GL Hypoxia-inducible factors: coupling glucose metabolism and redox regulation with induction of the breast cancer stem cell phenotype. The EMBO journal. 2017;36(3):252-259.

25. TIsty TD, Coussens LM Tumor stroma and regulation of cancer development. Annual review of pathology. 2006;1(unknow):119-150.

26. Mantovani A, Allavena P, Sica A, Balkwill F Cancer-related inflammation. Nature. 2008;454(7203):436-444. 
27. Aggarwal BB, Shishodia S, Sandur SK, Pandey MK, Sethi G Inflammation and cancer: how hot is the link? Biochemical pharmacology. 2006;72(11):16051621.

28. Batie M, Frost J, Frost M, Wilson JW, Schofield P, Rocha S Hypoxia induces rapid changes to histone methylation and reprograms chromatin. Science (New York, NY). 2019;363(6432):1222-1226.

29. Godet I, Shin YJ, Ju JA, Ye IC, Wang G, Gilkes DM Fate-mapping post-hypoxic tumor cells reveals a ROS-resistant phenotype that promotes metastasis. Nature communications. 2019;10(1):4862.

30. Palazon A, Goldrath AW, Nizet V, Johnson RS HIF transcription factors, inflammation, and immunity. Immunity. 2014;41(4):518-528.

31. Dai D, Chen B, Feng Y, Wang W, Jiang Y, Huang H, Liu J Prognostic value of prostaglandin 12 synthase and its correlation with tumor-infiltrating immune cells in lung cancer, ovarian cancer, and gastric cancer. Aging. 2020;12(10):9658-9685.

32. Pocha K, Mock A, Rapp C, Dettling S, Warta R, Geisenberger C, Jungk C, Martins LR, Grabe N, Reuss D et al Surfactant Expression Defines an Inflamed Subtype of Lung Adenocarcinoma Brain Metastases that Correlates with Prolonged Survival. Clinical cancer research : an official journal of the American Association for Cancer Research. 2020;26(9):2231-2243.

33. Echevarria MI, Awasthi S, Cheng CH, Berglund AE, Rounbehler RJ, Gerke TA, Takhar M, Davicioni E, Klein EA, Freedland SJ et al African American Specific Gene Panel Predictive of Poor Prostate Cancer Outcome. The Journal of urology. 2019;202(2):247-255.

34. Coussens LM, Werb Z Inflammation and cancer. Nature. 2002;420(6917):860-867.

35. El-Benna J, Hurtado-Nedelec M, Marzaioli V, Marie JC, Gougerot-Pocidalo MA, Dang PM Priming of the neutrophil respiratory burst: role in host defense and inflammation. Immunological reviews. 2016;273(1):180-193.

36. Murdoch C, Muthana M, Lewis CE Hypoxia regulates macrophage functions in inflammation. Journal of immunology (Baltimore, Md : 1950). 2005;175(10):6257-6263.

37. Saleh M, Trinchieri G Innate immune mechanisms of colitis and colitis-associated colorectal cancer. Nature reviews Immunology. 2011;11(1):9-20.

38. O'Connor PM, Lapointe TK, Beck PL, Buret AG Mechanisms by which inflammation may increase intestinal cancer risk in inflammatory bowel disease. Inflammatory bowel diseases. 2010;16(8):1411-1420.

39. Murdoch C, Giannoudis A, Lewis CE Mechanisms regulating the recruitment of macrophages into hypoxic areas of tumors and other ischemic tissues. Blood. 2004;104(8):2224-2234.

40. Makino Y, Yoon JH, Bae E, Kato M, Miyazawa K, Ohira T, Ikeda N, Kuroda M, Mamura M Repression of Smad3 by Stat3 and c-Ski/SnoN induces gefitinib resistance in lung adenocarcinoma. Biochemical and biophysical research communications. 2017;484(2):269-277.

41. Wang C, Jin S, Xu S, Cao S High Systemic Immune-Inflammation Index (SII) Represents an Unfavorable Prognostic Factor for Small Cell Lung Cancer Treated with Etoposide and Platinum-Based Chemotherapy. Lung. 2020;198(2):405-414.

42. Shi S, Yuan C, Zhuang K, Liang G, Yao Z, Wang D, Weng Q, Cao J, Luo P, Zhu H et al Resistance of SMMC-7721 hepatoma cells to etoposide in hypoxia is reversed by VEGF inhibitor. Molecular medicine reports. 2015;11(5):3842-3847.

43. Murakami A, Takahashi F, Nurwidya F, Kobayashi I, Minakata K, Hashimoto M, Nara T, Kato M, Tajima K, Shimada N et al Hypoxia increases gefitinibresistant lung cancer stem cells through the activation of insulin-like growth factor 1 receptor. PloS one. 2014;9(1):e86459.

44. Minakata K, Takahashi F, Nara T, Hashimoto M, Tajima K, Murakami A, Nurwidya F, Yae S, Koizumi F, Moriyama H et al Hypoxia induces gefitinib resistance in non-small-cell lung cancer with both mutant and wild-type epidermal growth factor receptors. Cancer science. 2012;103(11):1946-1954.

45. Wherry EJ, Kurachi M Molecular and cellular insights into T cell exhaustion. Nature reviews Immunology. 2015;15(8):486-499.

46. Cho H, Kang H, Lee HH, Kim CW Programmed Cell Death 1 (PD-1) and Cytotoxic T Lymphocyte-Associated Antigen 4 (CTLA-4) in Viral Hepatitis. International journal of molecular sciences. 2017;18(7):

47. Fourcade J, Sun Z, Benallaoua M, Guillaume P, Luescher IF, Sander C, Kirkwood JM, Kuchroo V, Zarour HM Upregulation of Tim-3 and PD-1 expression is associated with tumor antigen-specific CD8+ T cell dysfunction in melanoma patients. The Journal of experimental medicine. 2010;207(10):2175-2186.

48. Gao DN, Yang ZX, Qi QH Roles of PD-1, Tim-3 and CTLA-4 in immunoregulation in regulatory T cells among patients with sepsis. International journal of clinical and experimental medicine. 2015;8(10):18998-19005.

49. Harris-Bookman S, Mathios D, Martin AM, Xia Y, Kim E, Xu H, Belcaid Z, Polanczyk M, Barberi T, Theodros D et al Expression of LAG-3 and efficacy of combination treatment with anti-LAG-3 and anti-PD-1 monoclonal antibodies in glioblastoma. International journal of cancer. 2018;143(12):3201-3208.

50. Haymaker C, Wu R, Bernatchez C, Radvanyi L PD-1 and BTLA and CD8(+) T-cell "exhaustion" in cancer: "Exercising" an alternative viewpoint. Oncoimmunology. 2012;1(5):735-738.

51. Zhang C, Shao Y, Yang S, Bi X, Li L, Wang H, Yang N, Li Z, Sun C, Li L et al T-cell tolerance and exhaustion in the clearance of Echinococcus multilocularis: role of inoculum size in a quantitative hepatic experimental model. Scientific reports. 2017;7(1):11153.

52. Wu L, Mao L, Liu JF, Chen L, Yu GT, Yang LL, Wu H, Bu LL, Kulkarni AB, Zhang WF et a/ Blockade of TIGIT/CD155 Signaling Reverses T-cell Exhaustion and Enhances Antitumor Capability in Head and Neck Squamous Cell Carcinoma. Cancer immunology research. 2019;7(10):1700-1713.

53. Yates KB, Tonnerre P, Martin GE, Gerdemann U, Al Abosy R, Comstock DE, Weiss SA, Wolski D, Tully DC, Chung RT et al Epigenetic scars of CD8(+) T cell exhaustion persist after cure of chronic infection in humans. Nature immunology. 2021;22(8):1020-1029.

54. Taniuchi I CD4 Helper and CD8 Cytotoxic T Cell Differentiation. Annual review of immunology. 2018;36(unknow):579-601.

55. Oh SA, Li MO TGF-B: guardian of T cell function. Journal of immunology (Baltimore, Md : 1950). 2013;191(8):3973-3979.

\section{Tables}


Hypoxia and immune related signaling pathways

GOMF_TRANSFORMING_GROWTH_FACTOR_BETA_BINDING

GOMF_T_CELL_RECEPTOR_BINDING

GOMF_COMPLEMENT_RECEPTOR_ACTIVITY

GOBP_T_CELL_ACTIVATION_VIA_T_CELL_RECEPTOR_CONTACT_WITH_ANTIGEN_BOUND_TO_MHC_MOLECULE_ON_ANTIGEN_PRESENTIN

GOBP_REGULATION_OF_NEUTROPHIL_ACTIVATION

GOBP_REGULATION_OF_DENDRITIC_CELL_ANTIGEN_PROCESSING_AND_PRESENTATION

GOBP_POSITIVE_REGULATION_OF_LEUKOCYTE_ADHESION_TO_VASCULAR_ENDOTHELIAL_CELL

GOBP_POSITIVE_REGULATION_OF_ACUTE_INFLAMMATORY_RESPONSE

GOBP_INTERLEUKIN_35_MEDIATED_SIGNALING_PATHWAY

GOBP_DENDRITIC_CELL_ANTIGEN_PROCESSING_AND_PRESENTATION

KEGG_LEUKOCYTE_TRANSENDOTHELIAL_MIGRATION

KEGG_CYTOKINE_CYTOKINE_RECEPTOR_INTERACTION

KEGG_JAK_STAT_SIGNALING_PATHWAY

KEGG_CHEMOKINE_SIGNALING_PATHWAY

KEGG_NATURAL_KILLER_CELL_MEDIATED_CYTOTOXICITY

KEGG_B_CELL_RECEPTOR_SIGNALING_PATHWAY

KEGG_INTESTINAL_IMMUNE_NETWORK_FOR_IGA_PRODUCTION

HALLMARK_TNFA_SIGNALING_VIA_NFKB

HALLMARK_INFLAMMATORY_RESPONSE

HALLMARK_IL6_JAK_STAT3_SIGNALING

HALLMARK_IL2_STAT5_SIGNALING

HALLMARK_TGF_BETA_SIGNALING

HALLMARK_ALLOGRAFT_REJECTION

HALLMARK_HYPOXIA

HALLMARK_INTERFERON_GAMMA_RESPONSE

HALLMARK_INTERFERON_ALPHA_RESPONSE

\section{Figures}


A

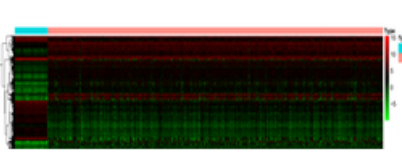

C

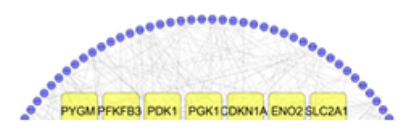

E

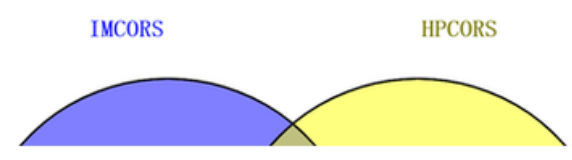

Figure 1

Screening of immune and hypoxia related genes (HMGS) in TCGA database. (A-B) Heat map (A) and volcano (B) map of significantly different genes. (C) PPI network of 200 hypoxia related genes. (D) Correlation matrix Heatmap of 49 anoxic core genes. (E) Take the intersection of hypoxia and immune related genes. (F-G) (F) KEGG and (G) GO functional enrichment analysis of $380 \mathrm{HMGs}$. 
A

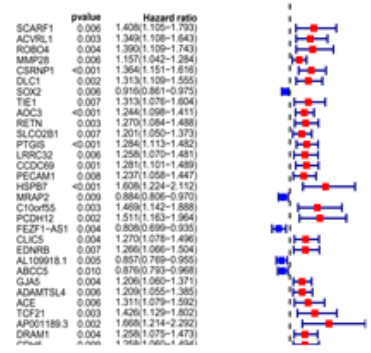

B

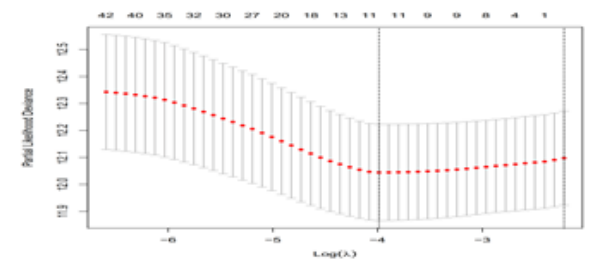

$\mathrm{C}$

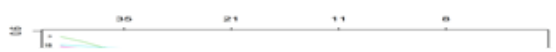

D

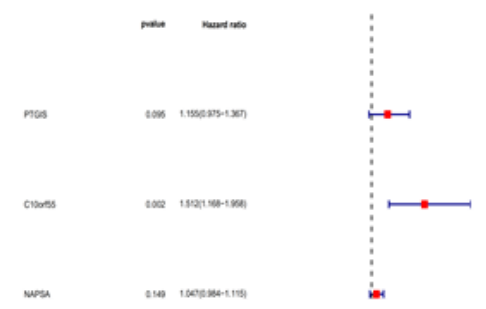

Figure 2

Establishment of immune and hypoxic prognosis model (HMPL). (A) The forest map shows the univariate Cox regression analysis of HMGs. (B) Ten-time cross-validation for tuning parameter selection in the least absolute shrinkage and selection operator (LASSO) model, (C) LASSO coefficient profiles of the 380 HMDGS. (D) Multivariate Cox regression analysis. (D) Principal component analysis (PCA) was used to identify the status of risk groups. (E) Kaplan-Meier and time-dependent ROC curves in the TCGA. $(\mathrm{H}-\mathrm{I})$ Box plot $(\mathrm{H})$ and heatmap $(\mathrm{I})$ show the expression level of risk genes in high and low risk groups. $(\mathrm{J})$ The correlation circle chart shows the correlation between risk score and risk model genes.

\section{Figure 3}

Effects of risk score and clinicopathological features on the prognosis of lung adenocarcinoma. (A-C) (A) Univariate, (B) multivariate Cox regression and (C) ROC curve analysis of risk score and multiple clinicopathological features. (D) Nomogram shows the prognostic value of risk score and pathological factors in patients with lung adenocarcinoma. (E) Calibration plot was used to estimate the accuracy of nomogram prediction ability. (H) Survival difference of high and low risk groups under different clinicopathological characteristics. 
A

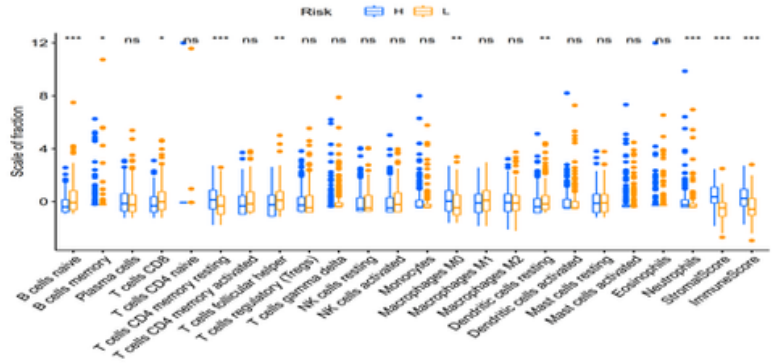

B

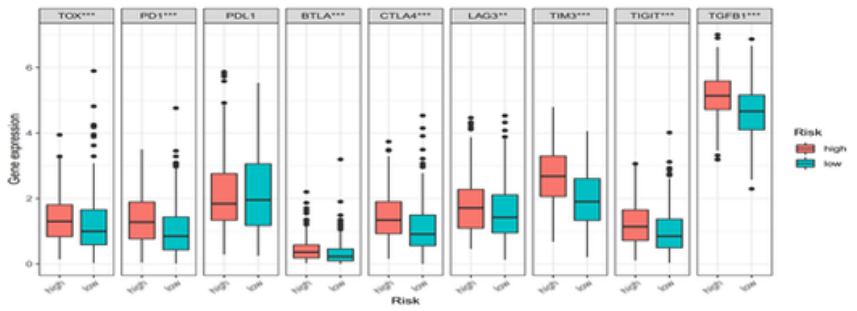

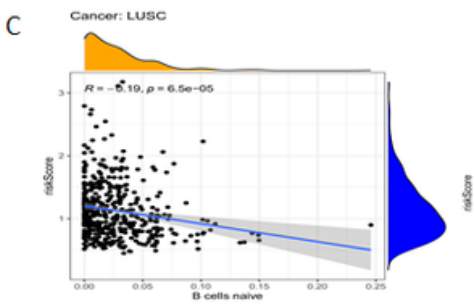
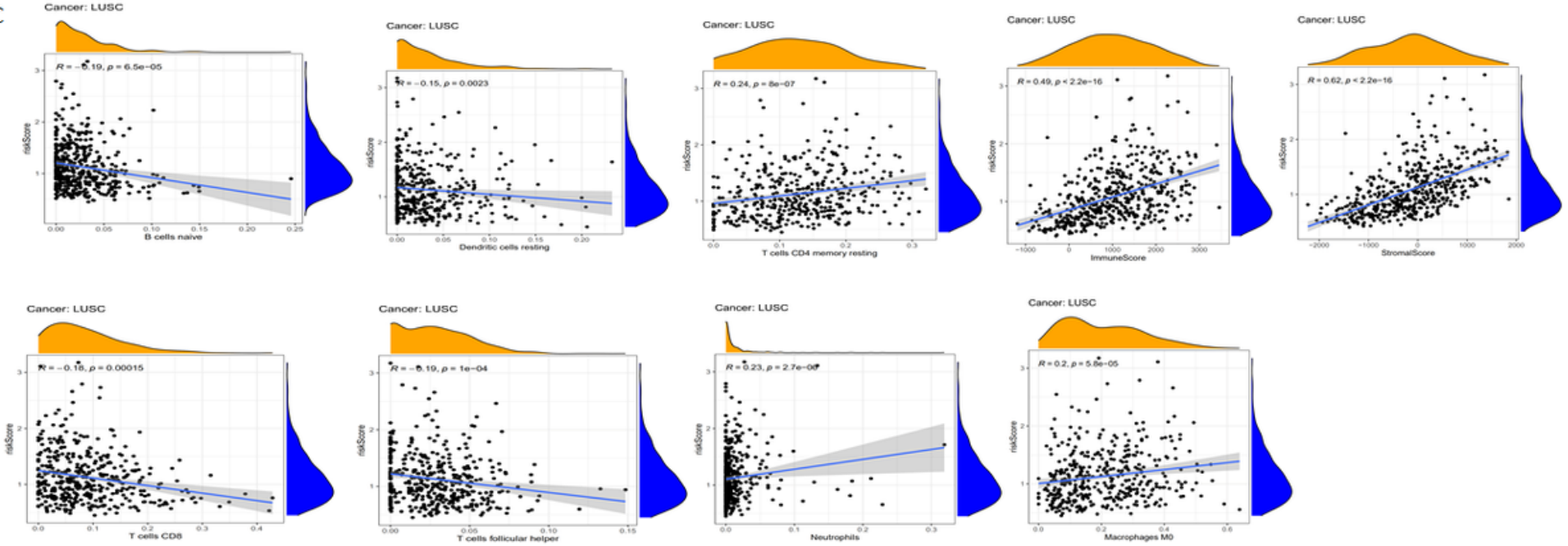

Figure 4

Immune cell infiltration in different risk groups. (A) Differences in the distribution of immune cells between high and low risk groups. (B) Distribution level of immune related genes in different risk groups. (C) Correlation level between immune cells and risk score.

A

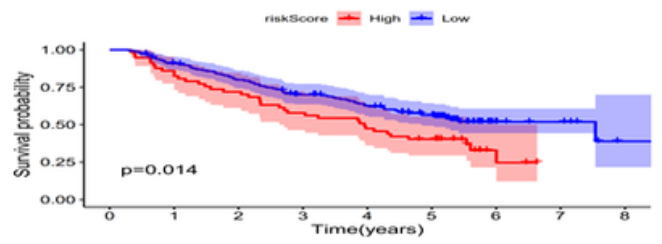

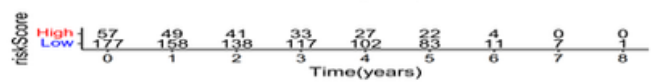

C

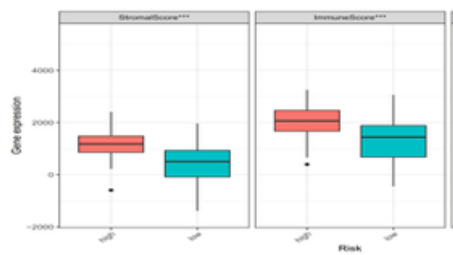

E
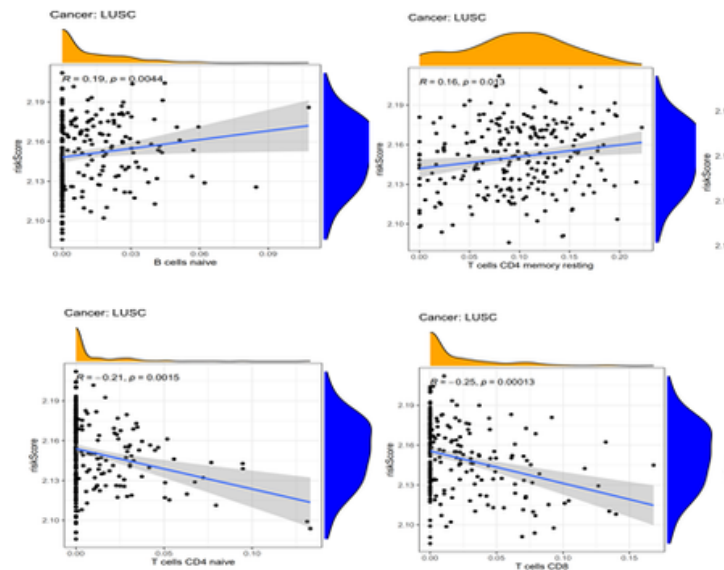

B

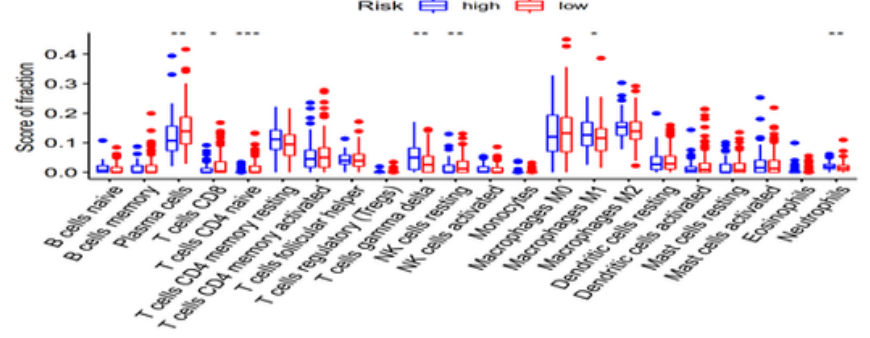

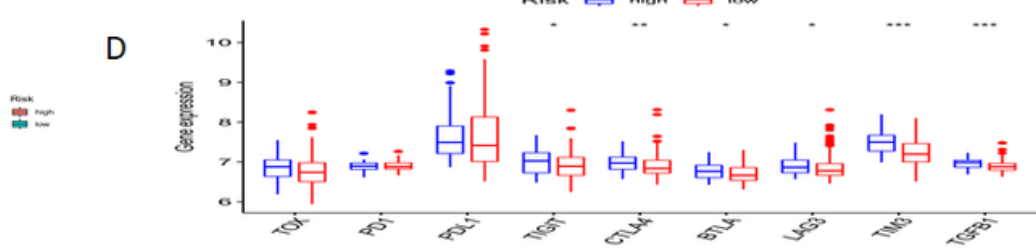
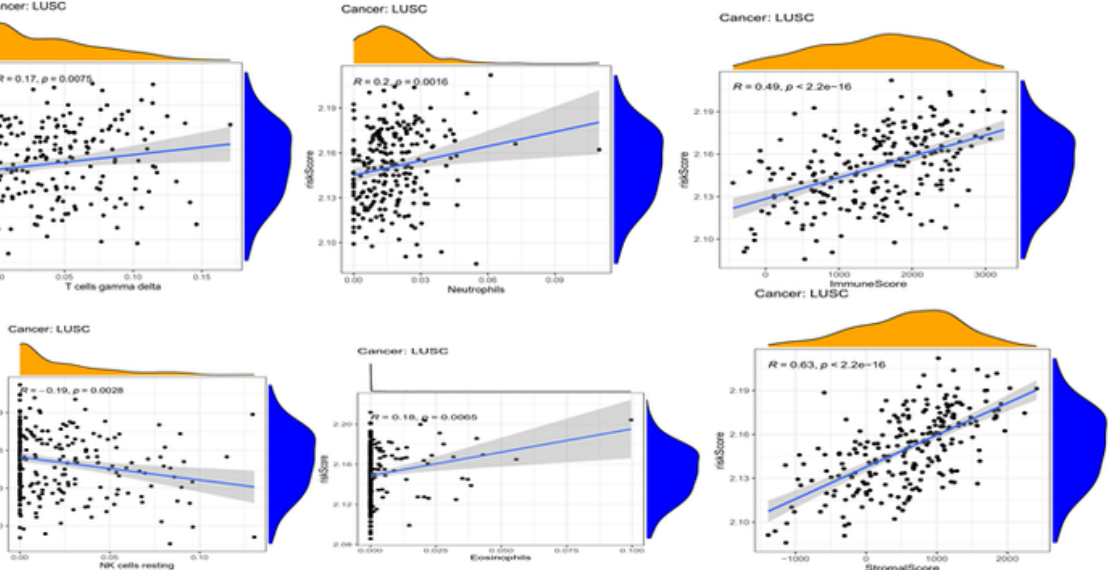

Page 13/15 
Figure 5

Differences of immune microenvironment between high and low risk groups based on GEO validation. (A) Survival differences between high and low risk groups. (B-C) (B) Immune cell distribution and (C) immune microenvironment scoring under different risk groups. (D) Distribution levels of different immune related genes in different risk groups. (E) Correlation level between immune cell score and risk score.

A

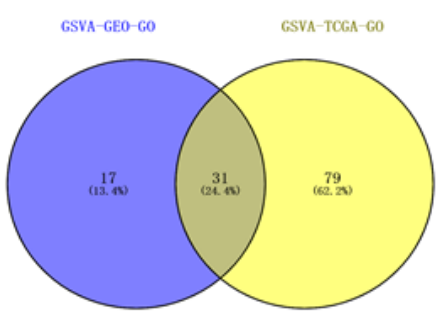

D

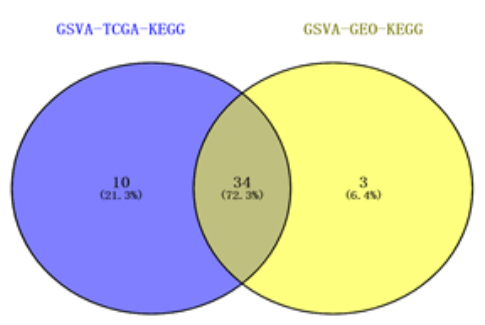

G

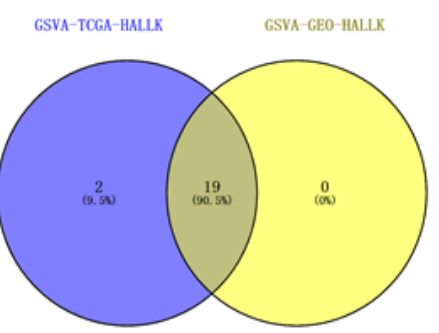

B
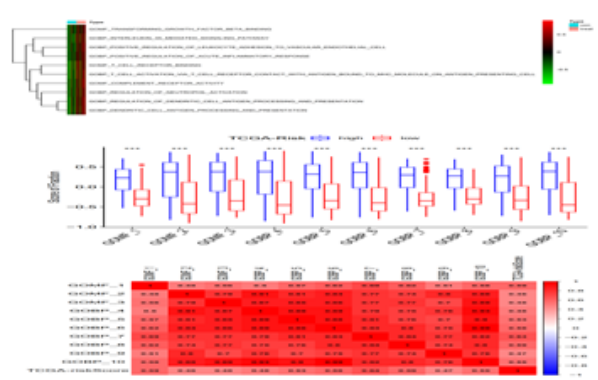

$\mathrm{E}$
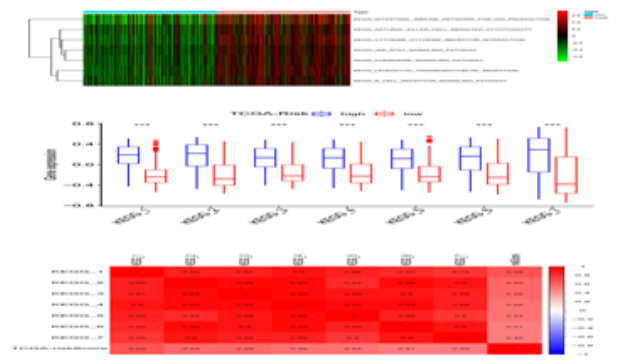

$\mathrm{H}$
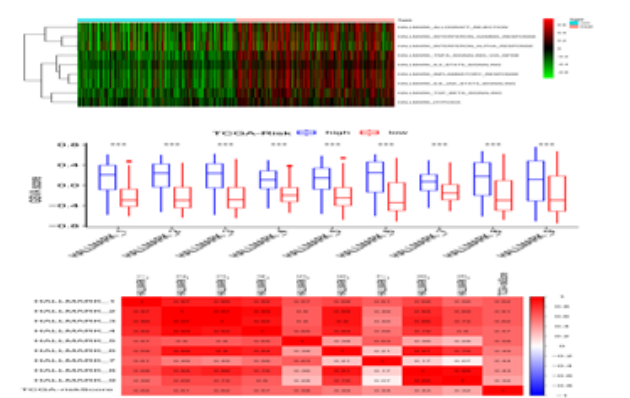

C
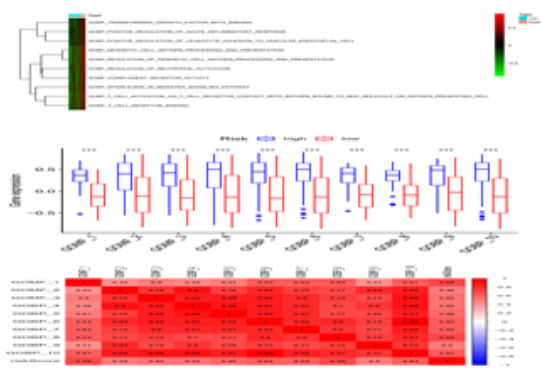

$\mathrm{F}$
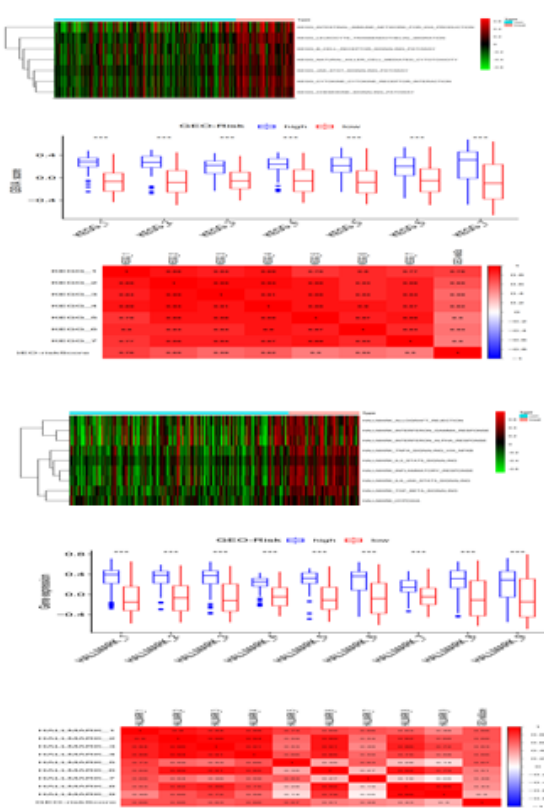

Figure 6

Gene set variation analysis (GSA) was performed on high-risk and low-risk groups based on the go, KEGG, and hallmark data sets. (a, D, g) signal pathways significantly enriched in high-risk groups in TCGA experimental group and geo test group were intersected with each other. (B, C, e, F, h, I) gsva analysis based on the go (B-C), KEGG (E-F), and hallmark (H-I) data sets. TCGA training sets $(B, e, H)$ and geo $(C, F, I)$ The high-risk group of the test set was mainly enriched in immune and hypoxia-related pathways (GO: 1-TRANSFORMING_GROWTH_FACTOR_BETA_BINDING, 2-T_CELL_RECEPTOR_BINDING, 3-

COMPLEMENT_RECEPTOR_ACTIVITY, 4-

T_CELL_ACTIVATION_VIA_T_CELL_RECEPTOR_CONTACT_WITH_ANTIGEN_BOUND_TO_MHC_MOLECULE_ON_ANTIGEN_PRESENTING_CELL, 5REGULATION_OF_NEUTROPHIL_ACTIVATION, 6-REGULATION_OF_DENDRITIC_CELL_ANTIGEN_PROCESSING_AND_PRESENTATION, 7-

POSITIVE_REGULATION_OF_LEUKOCYTE_ADHESION_TO_VASCULAR_ENDOTHELIAL_CELL, 8-

POSITIVE_REGULATION_OF_ACUTE_INFLAMMATORY_RESPONSE, 9-GOBP_INTERLEUKIN_35_MEDIATED_SIGNALING_PATHWAY, 10DENDRITIC_CELL_ANTIGEN_PROCESSING_AND_PRESENTATION. KEGG: 1-LEUKOCYTE_TRANSENDOTHELIAL_MIGRATION, 2-

CYTOKINE_CYTOKINE_RECEPTOR_INTERACTION, 3-JAK_STAT_SIGNALING_PATHWAY, 4-CHEMOKINE_SIGNALING_PATHWAY, 5B_CELL_RECEPTOR_SIGNALING_PATHWAY, 6-NATURAL_KILLER_CELL_MEDIATED_CYTOTOXICITY, 7-

INTESTINAL_IMMUNE_NETWORK_FOR_IGA_PRODUCTION. HALLK: 1-TNFA_SIGNALING_VIA_NFKB, 2-INFLAMMATORY_RESPONSE, 3IL6_JAK_STAT3_SIGNALING, 4-IL2_STAT5_SIGNALING, 5-TGF_BETA_SIGNALING, 6-ALLOGRAFT_REJECTION, 7-HYPOXIA, 8INTERFERON_GAMMA_RESPONSE, 9-INTERFERON_ALPHA_RESPONSE.)

\section{Figure 7}

Under TCGA and geo data sets, the chemotherapeutic effects of different risk groups. (A) Gemcitabine. (B) Gefitinib. (C) Etoposide. (D) Doxorubicin. (E) Docetaxel. (F) Cisplatin. (G) Bleomycin. 
A

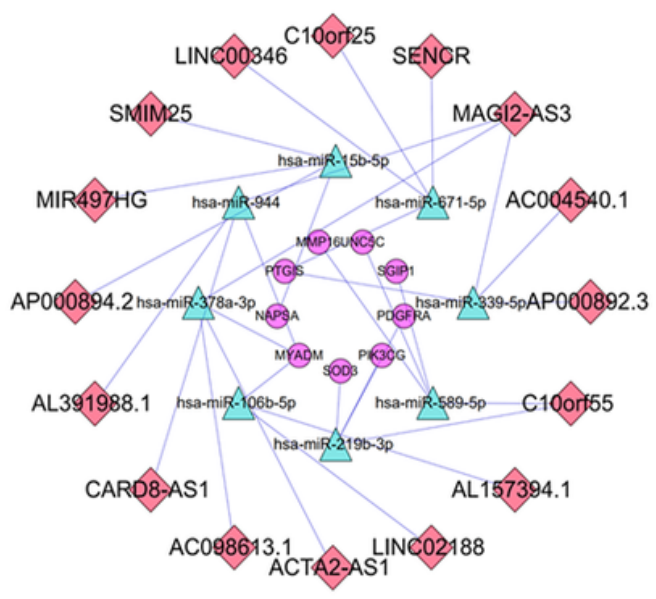

B

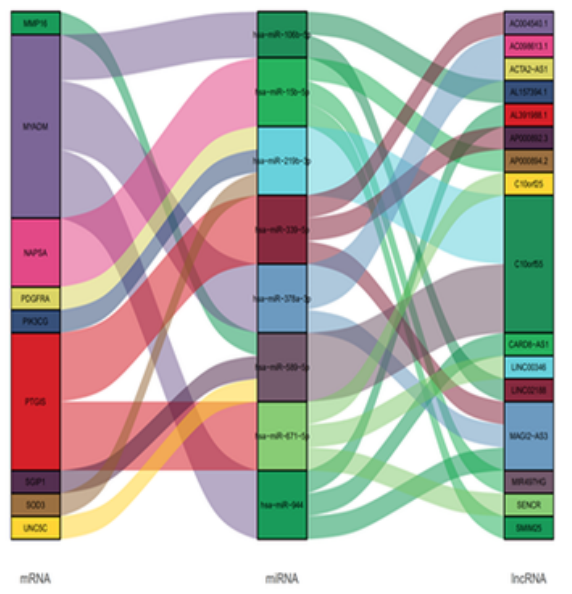

C

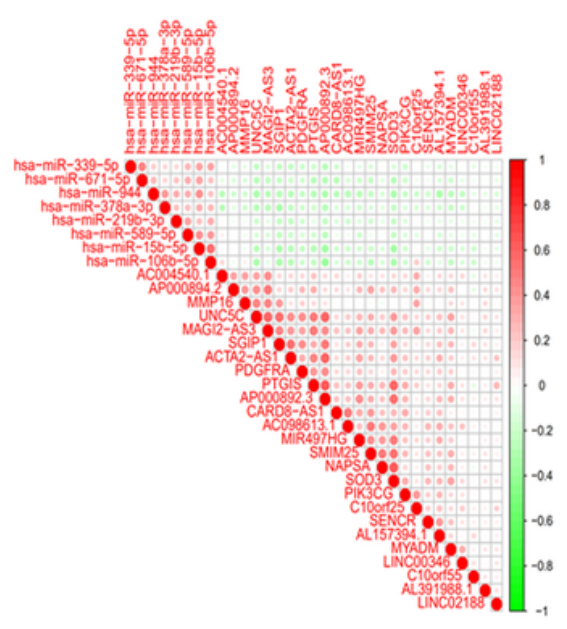

Figure 8

establish a CeRNA network related to risk genes. (A) install Cerna network diagram. (B) Sankey diagram shows the relationship between IncRNA miRNA mRNA in Cerna. (C) the correlation matrix heat map shows the regulatory relationship among the IncRNA, miRNA, and mRNA.

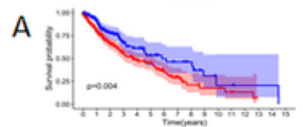

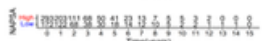

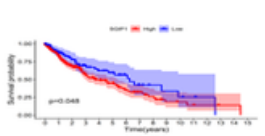

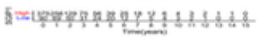
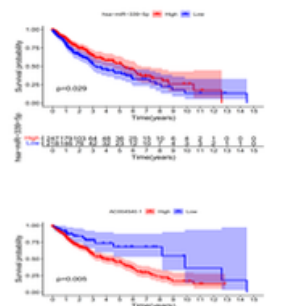

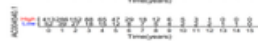

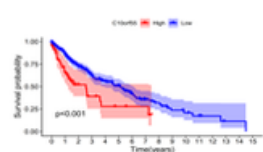

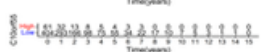

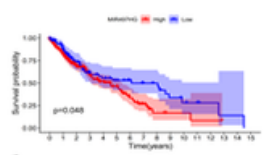

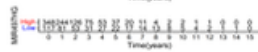

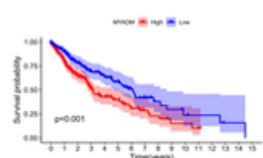

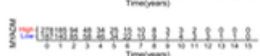

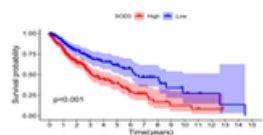

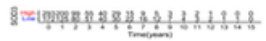
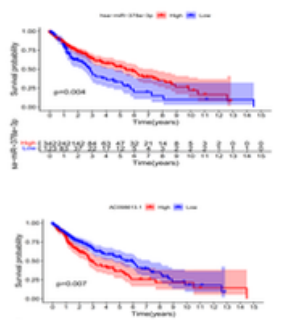

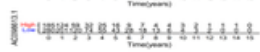
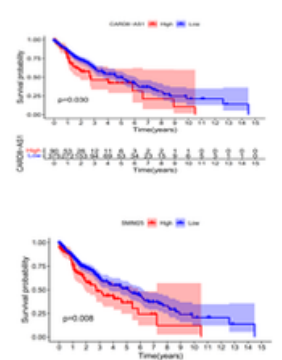

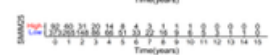

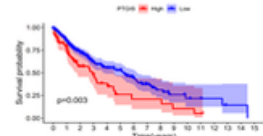

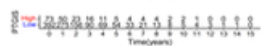

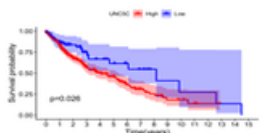

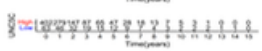
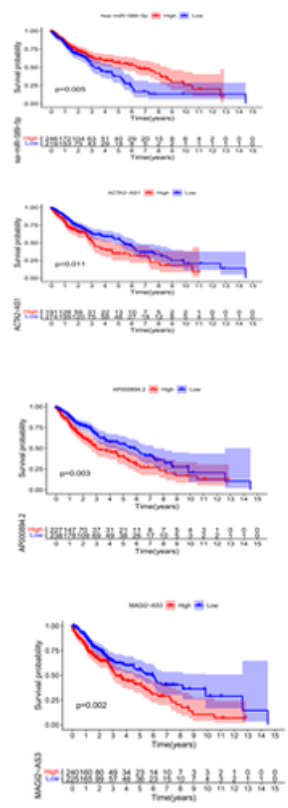

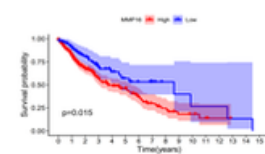

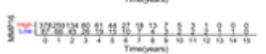

B
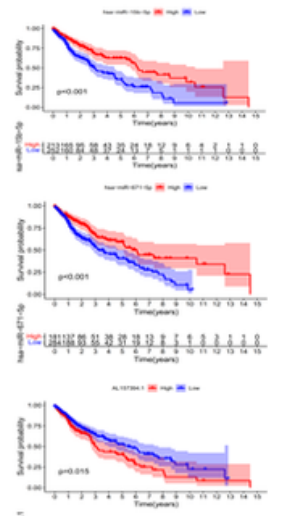

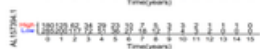

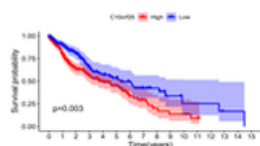

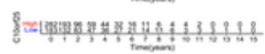
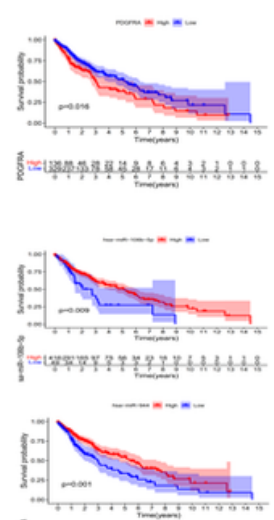

F)

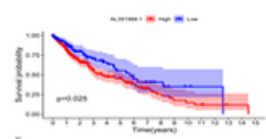

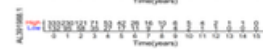

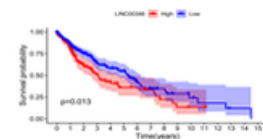

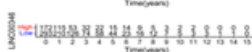
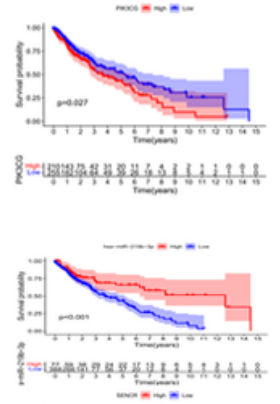

C

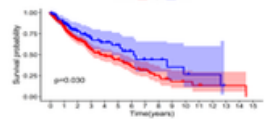

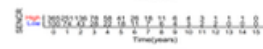

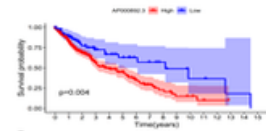

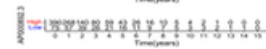

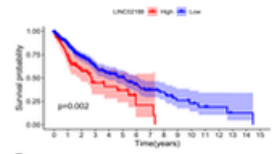

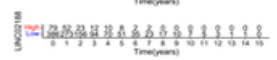

\section{Figure 9}

Survival analysis of Cerna network-related genes. (A) mRNA. (B) miRNA. (C) InCRNA. 